\title{
CrystEngComm
}

Q1

10

Cite this: DOI: 10.1039/c4ce00192c

\section{Evaluation of crystallization kinetics of adipic acid in an oscillatory baffled crystallizer}

\author{
C. J. Brown, ${ }^{a}$ Y. C. Lee, ${ }^{\text {b }}$ Z. K. Nagy ${ }^{c}$ and X. $\mathrm{Ni}^{\mathrm{a}}$
}

For solution crystallization, nucleation can be characterized by the maximum sub-cooling (or metastable limit), which is known to vary with numerous process parameters. The relationship between the metastable limit and cooling rate is of particular interest, as it can be utilized to derive nucleation kinetic parameters. However, this relationship is open to interpretation. This work presents the application of three such interpretations (Nývlt, Kubota and a population balance based method) to a cooling crystallization of adipic acid in an oscillatory baffled crystallizer, a relatively new type of crystallizer with increased studies and applications in continuous plug flow operation. It also considers the role the device employed to detect nucleation events plays in the derived kinetic parameters. The result of this study shows that although all three interpretations can reasonably predict the maximum sub-cooling over a tested range of cooling rates, the linear assumptions in the Nývlt and Kubota interpretations give increased deviations from the experimental data, in particular for faster cooling rates. In contrast to the two aforementioned models, the population balance based method maintains a minimal deviation across the whole range of cooling rates used. In addition, although the population balance method does not consider the sensitivity of detection tools in its implementation, while the Kubota method does, the sensitivity of nucleation detection is reflected in the derived nucleation rate constants.
Received 24th January 2014, Accepted 14th July 2014

DOI: $10.1039 /$ c4ce00192c

www.rsc.org/crystengcomm long been established that, due to the time required for the critical nuclei to grow to a detectable size, the technique used for detecting $\Delta T_{\max }$ has an influence on the resulting estimated kinetics. ${ }^{1,14}$ For example, Fujiwara and co-workers ${ }^{6}$ compared focused beam reflectance measurements (FBRM) to those from ATR-FTIR spectroscopy and concluded that FBRM was more sensitive than both the ATR-FTIR and visual observations. In addition to this work, Parsons and co-workers ${ }^{10}$ evaluated turbidity measurements, using a range of gains, against visual observations from an operator and found that the operator consistently detected the presence of nuclei before the turbidity meter. Simon and co-workers ${ }^{7}$ assessed external bulk video imaging (akin to visual observations) over FBRM and UV-Vis spectroscopy and found all three to have comparable performance in detecting the onset of nucleation.

Recently, Gherras and Fevotte ${ }^{14}$ compared four of the more widely used theoretical approaches ${ }^{2-4,9}$ of $\Delta T_{\text {max }}$. While all approaches predicted the experimental values well, due to growth rate and potentially second nucleation playing a role in detection of nucleation, only relative and partial information on nucleation kinetics was obtained, regardless of detection device sensitivity. Therefore, they suggested ${ }^{14}$ that the application of population balance equations (PBE) would yield significant improvements, which was implemented in the follow up work ${ }^{15}$ by the same authors. In a result comparable to the simplified $\mathrm{PBE}$ method used previously, ${ }^{16}$ their 
study ${ }^{15}$ demonstrated that both an experimentally measured concentration and crystal size distribution (CSD) were required for the separation of nucleation and growth parameters. Gherras and Fevotte ${ }^{14}$ also concluded that despite deriving a rough and approximate CSD from in situ imaging, the distribution did provide sufficient information to allow quantification of the nucleation kinetics. This success has led to the potential applications of other image processing techniques ${ }^{17}$ to supplement concentration data for parameter estimation.

For the first time, this manuscript presents kinetic parameter estimations for crystallization of adipic acid in an oscillatory baffled crystallizer, a crystallizer which potentially has a different nucleation mechanism ${ }^{18,19}$ to stirred tank crystallizers used in previous works. ${ }^{4,14,16}$ The work presented here also aims to supplement previous studies ${ }^{6,7,10}$ by comparing a non-intrusive process video imaging (PVI) detection technique with a traditional turbidity probe and to further the evaluation of three theoretical interpretations of $\Delta T_{\max }$ in the oscillatory baffled crystallizer.

\section{Theory}

\section{Method 1: Nývlt approach}

A full derivation for Nývlt interpretation can be found elsewhere $^{2,3}$ but in essence it relates the maximum sub-cooling, $\Delta T_{\max }$, with the cooling rate, $\beta$, based on the assumption that the nucleation rate, $B$, is equal to the rate of supersaturation generation at the onset of nucleation, eqn (1), resulting in the linear relationship of eqn (2).

$$
\begin{gathered}
B=\frac{\mathrm{d} \Delta C}{\mathrm{~d} t} \\
\ln \beta=b \ln \left(\Delta T_{\max }\right)+\ln k_{\mathrm{b}}^{\prime}+(b-1) \ln \left(\frac{\mathrm{d} C_{\mathrm{sat}}}{\mathrm{d} T}\right)
\end{gathered}
$$

where $b$ and $k^{\prime}{ }_{\mathrm{b}}$ are the nucleation order and the mass rate constant respectively, $\mathrm{d} C_{\text {sat }} / \mathrm{d} T$ is the slope of the solubility curve for a given saturation temperature. From eqn (2) a straight line would be expected when plotting $\ln \beta$ versus $\ln$ $\Delta T_{\max }$, with a gradient equal to the apparent nucleation order, $b$. The mass nucleation rate constant, $k^{\prime}{ }_{b}$, is determined from the intercept. The mass rate constant is then converted to a number based rate constant by the following:

$$
k_{\mathrm{b}}=\frac{k_{\mathrm{b}}^{\prime}}{\alpha \rho_{\mathrm{c}} r^{3}}
$$

where $k_{\mathrm{b}}$ is the number nucleation rate constant, $\alpha$ the volume shape factor, $\rho_{\mathrm{c}}$ the crystal density and $r$ the average nuclei size.

\section{Method 2: Kubota approach}

Similar to the Nývlt approach, a detailed derivation of the Kubota interpretation can also be found elsewhere. ${ }^{1,20}$ The most significant difference between the Nývlt and the Kubota approaches is that the Kubota interpretation equates the nucleation rate, $B$, to the rate of the change of a detected nuclei density, $N / V$, with time:

$$
B=\frac{\mathrm{d}(N / V)}{\mathrm{d} t}
$$

Resulting in a linear relationship of the form:

$$
\ln \Delta T_{\max }=\frac{1}{b+1} \ln \left[\left(\frac{N_{\mathrm{m}}}{V k_{\mathrm{b}}^{\prime}}\right)(b+1)\right]+\frac{1}{b+1} \ln \beta
$$

where $N_{\mathrm{m}} / V$ is the minimum detectable number density of the nuclei and is apparatus dependant. From eqn (5) it suggests that a plot of $\ln \Delta T_{\max }$ versus $\ln \beta$ would yield a straight line, with a gradient equal to $1 /(b+1)$, where $b$ is the nucleation order. The mass nucleation rate constant, $k_{\mathrm{b}}^{\prime}$, could then be determined from the intercept. Similarly to the Nývlt interpretation, the number based nucleation rate constant is also calculated from eqn (3).

\section{Method 3: Population Balance (PB) approach}

As mentioned, previous studies ${ }^{15,16}$ have highlighted that the classical approaches (methods 1 and 2) encompass several assumptions which affect the accuracy of the determined kinetic parameters. These assumptions can be avoided by describing the crystallization process as a moment transform in a simplified PBE. ${ }^{21}$ When only a one dimensional characteristic size is considered, the $j$ th moment can be defined as:

$$
\mu_{j}=\int_{0}^{\infty} L^{j} f_{\mathrm{n}}(L, t) \mathrm{d} L
$$

where $f_{\mathrm{n}}(L, t)$ is the crystal size distribution, $t$ is the time and $L$ is the characteristic crystal size. Therefore, a complete model of the crystallization process can be described by considering the first four moment equations and the mass balance equation ${ }^{16}$ as:

$$
\left[\begin{array}{c}
\mathrm{d} \mu_{0} / \mathrm{d} t \\
\mathrm{~d} \mu_{1} / \mathrm{d} t \\
\mathrm{~d} \mu_{2} / \mathrm{d} t \\
\mathrm{~d} \mu_{3} / \mathrm{d} t \\
\mathrm{~d} C / \mathrm{d} t
\end{array}\right]=\left[\begin{array}{c}
B \\
G \mu_{0}+B r_{0} \\
2 G \mu_{1}+B r_{0}{ }^{2} \\
3 G \mu_{2}+B r_{0}{ }^{3} \\
-\rho_{\mathrm{c}} k_{\mathrm{v}}\left(3 G \mu_{2}+B r_{0}{ }^{3}\right)
\end{array}\right]
$$

where $C$ is the solution concentration ( $\mathrm{g}$ per $\mathrm{g}$ solvent), $r_{0}$ is the crystal size at nucleation, $\rho_{\mathrm{c}}$ the crystal density $\left(1.36 \mathrm{~g} \mathrm{ml}^{-1}\right.$ for adipic acid), ${ }^{22} k_{\mathrm{v}}$ the volume shape factor $(\pi / 6)^{23}$ and $B$ and $G$ are the primary nucleation and growth rates respectively, which are described by the power law expressions:

$$
\begin{aligned}
& B=k_{\mathrm{b}} \Delta C^{\mathrm{b}} \\
& G=k_{\mathrm{g}} \Delta C^{\mathrm{g}}
\end{aligned}
$$

In addition the solubility of adipic acid ${ }^{24,25}$ ( $\mathrm{g} \mathrm{AA} \mathrm{g}^{-1}$ water) as a function of temperature (in ${ }^{\circ} \mathrm{C}$ ) is defined by: 


$$
C_{\text {sat }}(T)=0.0059 \mathrm{e}^{0.0545 T}
$$

For unseeded systems, the initial conditions for eqn (7) are defined as: $\mu_{i}(0)=0(i=0,1,2,3)$ and $C(0)=C_{i}$. Moreover, the size of the nuclei is considered negligible $\left(r_{0}=0\right) .{ }^{16}$

The non-linear moments and the concentration equation set, as given in eqn (7), can be solved using a fast and efficient globally convergent Newton-Raphson solver in order to carry out multi-dimensional root finding solutions by minimising the equations while avoiding the solution descending into a local minimum. Details of the numerical methodology are available in the work by Gaskell and co-workers. ${ }^{26}$ Using the experimental data for the maximum sub-cooling, $\Delta T_{\max }$, and the cooling rate, $\beta$, along with appropriate values for the kinetic parameters, $k_{\mathrm{b}}, b, k_{\mathrm{g}}$ and $g$, result in the computed solutions for concentration, $C$, and the moments, $\mu_{0}, \mu_{1}, \mu_{2}$, and $\mu_{3}$. To determine the maximum sub-cooling temperature, $T$, at which nucleation occurs, the solver is iterated over time interval, $0<t<t_{\max }$, and the solution is chosen when supersaturation, $\Delta C$, is at its maximum.

$$
\min _{\theta}\left\{\mathrm{obj}=\sum_{i=1}^{N_{\beta}}\left|\Delta T_{\max , i}(\theta, \beta)-\Delta T_{\max , i}^{\exp }(\beta)\right|^{2}\right\}
$$

The kinetic parameters $\left(k_{\mathrm{b}}, b, k_{\mathrm{g}}\right.$ and $\left.g\right)$ are estimated by performing a non-linear optimisation via an iterative scattering approach (minimisation of mean squared error between the experimental and calculated maximum sub-coolings was utilized as the objective as given by eqn (11), where $\theta=\left(k_{\mathrm{b}}, b\right.$, $k_{\mathrm{g}}, g$ ) and $N_{\beta}$ the number of cooling rates) in tandem with the globally convergent Newton-Raphson solver. A pre-defined number of randomly generated kinetic parameter sets, within prescribed lower and upper bounds, are used in each of the scattering process where successive iterations optimise the kinetic parameters and narrow down the limits in which it operates to ensure that the supersaturation concentration, $\Delta C$, is always at its maximum. The iterative scattering process is repeated until the optimised accuracy of $\Delta C$ is within a pre-defined tolerance.

\section{Experimental methods}

\section{Experimental setup}

The apparatus utilized in the work presented here was consisted of a jacketed $1000 \mathrm{ml}$ (40 mm internal diameter) oscillatory baffled crystallizer (OBC), with a working volume of $400 \mathrm{ml}$. A further square cross section jacket was fitted to the exterior of the OBC and filled with water. This second jacket corrected for the curvature of the column when taking optical measurements. Oscillation was applied through a nitrile diaphragm driven by an electric motor mounted beneath the OBC. Both amplitude and frequency of oscillation were kept constant throughout all experiments at $15 \mathrm{~mm}$ peak to trough and $0.9 \mathrm{~Hz}$, respectively. Heating/cooling was provided to the OBC through the interior jacket by an external water bath and pump (grant GP200R2). Illumination for the camera was provided by a continuous $1 \mathrm{~W}$ argon ion laser (Spectra Physics) split into two light sheets, $2.31 \mathrm{~mm}$ thick by $60 \mathrm{~mm}$ wide. Both sides of the OBC were illuminated through the centre to provide a uniform light distribution. Images from the CCD camera (The ImagingSource) were recorded directly to the PC hard drive at $10 \mathrm{~s}$ intervals. Exposure time for the images was constant at $1 / 2000 \mathrm{~s}$. In addition to the camera setup, a turbidity probe and thermocouple (HEL Group Ltd) were also placed into the OBC. Again the output of these was logged directly to the PC at $10 \mathrm{~s}$ intervals. A cross sectional drawing of the $\mathrm{OBC}$ and a schematic of the setup are shown in Fig. 1.

\section{Experimental materials and methods}

An aqueous solution, saturated at $40^{\circ} \mathrm{C}$, of adipic acid (AA) (Fisher Scientific 99\% m.p. $152^{\circ} \mathrm{C}$ to $154^{\circ} \mathrm{C}$ ) was produced by adding $20.25 \mathrm{~g}$ of $\mathrm{AA}$ to $400 \mathrm{~g}$ of distilled water $\left(C_{i}=\right.$ $0.051 \mathrm{~g} \mathrm{~g}^{-1}$ ) and heating it to $60{ }^{\circ} \mathrm{C}$ on a hot plate; mixing was achieved through a magnetic stirrer bar. The solution was cured at this temperature for $30 \mathrm{~min}$ to ensure complete dissolution. Hot solution was then hot filtered through a $1.2 \mu \mathrm{m}$ glass fibre filter to remove any foreign bodies. The hot filtered solution was added to the pre-warmed $\left(50{ }^{\circ} \mathrm{C}\right)$ OBC before oscillation was applied. Prior to imaging, the light sheets were aligned and the camera positioned perpendicular to them. Camera lens and the region of interest were then adjusted to ensure that the camera was focused on an area of $11.37 \mathrm{~mm} \times 12.52 \mathrm{~mm}$ between a pair of baffles. Cooling to $30{ }^{\circ} \mathrm{C}$ was applied at a fixed linear rate outlined in Table 1. Images from the CCD camera and data from the turbidity probe and thermocouple were recorded every $10 \mathrm{~s}$ during the experimental time. The solution was then matured at $30^{\circ} \mathrm{C}$ for $1 \mathrm{hr}$ before heating it back to $50{ }^{\circ} \mathrm{C}$ at a

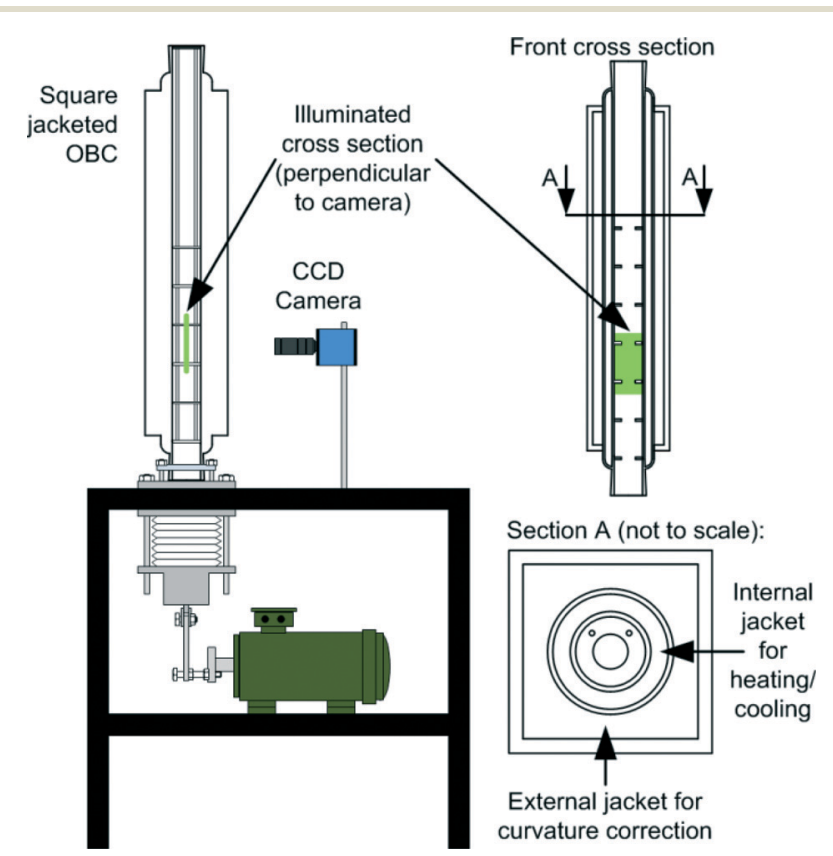

Fig. 1 Experimental setup showing cross sectional drawing of $O B C$ with internal and external jackets. 
Table 1 Experimental conditions and measured data

\begin{tabular}{|c|c|c|c|c|}
\hline \multirow[b]{2}{*}{$\operatorname{Exp}$} & \multirow[b]{2}{*}{$\beta\left({ }^{\circ} \mathrm{C} \min ^{-1}\right)$} & Turbidity & MGV & \multirow{2}{*}{$\frac{\mathrm{MGV}}{L_{\mathrm{d}}(\mu \mathrm{m})}$} \\
\hline & & $\Delta T_{\max }\left({ }^{\circ} \mathrm{C}\right) \pm 0.15$ & $\Delta T_{\max }\left({ }^{\circ} \mathrm{C}\right) \pm 0.15$ & \\
\hline A & 1.67 & 5.66 & 7.88 & $14.41 \pm 12.50$ \\
\hline B & 0.64 & 4.06 & 5.80 & $15.65 \pm 17.91$ \\
\hline $\mathrm{C}$ & 0.44 & 2.51 & 4.35 & $28.98 \pm 40.56$ \\
\hline D & 0.22 & 1.12 & 1.67 & $13.37 \pm 21.41$ \\
\hline $\mathrm{E}$ & 0.23 & 1.26 & 1.42 & $14.41 \pm 12.61$ \\
\hline $\mathrm{F}$ & 0.10 & 0.65 & 1.37 & $14.88 \pm 12.80$ \\
\hline
\end{tabular}

\section{Results and discussion}

Table 1 shows the experimental conditions, the maximum sub-cooling obtained from both the turbidity and MGV measurements as well as the mean crystal size $\left(L_{\mathrm{d}}\right)$ determined experimentally from the image analysis at the point nuclei were first detected (note that the mean crystal size cannot be acquired from the turbidity measurement alone). It would be expected that the faster the cooling rate, the smaller the

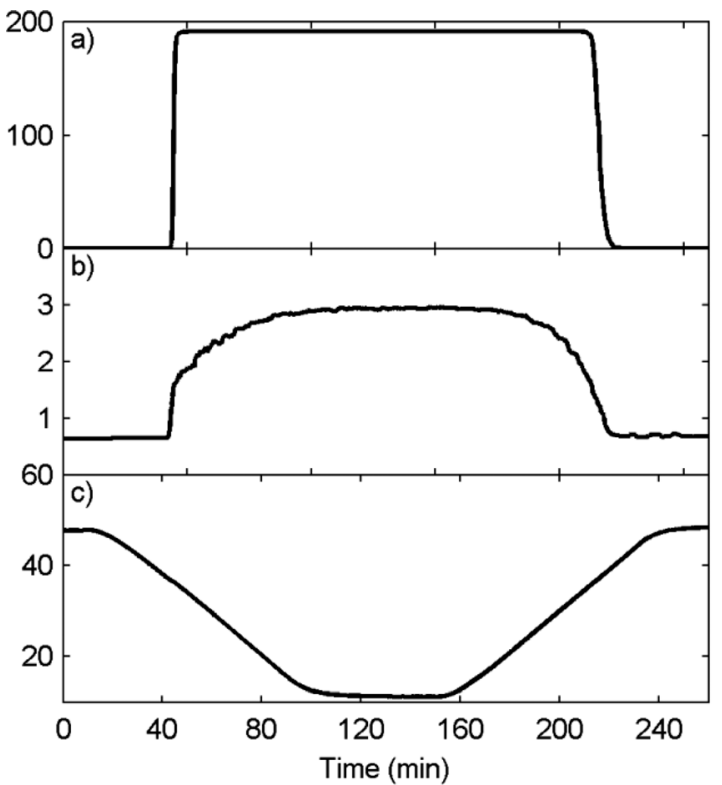

Fig. 2 Typical measured a) MGV, b) turbidity (V) and c) temperature $\left({ }^{\circ} \mathrm{C}\right)$.

detected nuclei size. ${ }^{16}$ However, this trend is not consistent with the data in Table 1, where the observed mean particle size is approximately the same value $(\approx 14 \mu \mathrm{m})$ for all experiments, suggesting that the minimum detectable particle size was limited by the capability of the image capture setup. This is in line with the previously reported minimum detectable particle sizes for this type of image capture systems. ${ }^{17,27}$

The data in Table 1 also show that the $\Delta T_{\max }$ values from the MGV measurements are greater than those from the turbidity measurements across all cooling rates. Kubota ${ }^{1}$ previously proposed that the more sensitive the nucleation detection device, the narrower the observed metastable limit, and quantified the sensitivity in the form of the minimum detectable number density $N_{\mathrm{m}} / V$. The results shown in Table 1 would suggest that, in comparison to the MGV arrangement, the turbidity probe requires a lower density of crystals present to give a positive indication of nucleation. This conclusion is contrary to the calculated minimum detectable number density values, which rank the MGV arrangement as the more sensitive technique (see Appendix 1 for calculations).

Utilizing the data from Table 1 , the estimated kinetic parameters for both techniques from all three models are summarized in Table 2 . Note that methods 1 and 2 only deal with the nucleation kinetics ( 2 parameter fit), while method 3 predicts kinetics of both nucleation and growth (4 parameter fit). A closer look at the kinetic parameters in Table 2 reveals that there is no statistical difference between the turbidity and MGV techniques for the estimation of these parameters using method 1 , where the sensitivity of the measuring tools for detecting nucleation was not considered. As would be expected, the inclusion of such sensitivity in method 2 results in a larger difference in the kinetic parameters, $k_{\mathrm{b}}$ and $b$, between the two detection techniques.

Although there is no statistical difference between the growth order, $g$, and the growth rate constant, $k_{\mathrm{g}}$, for the turbidity and MGV techniques by method 3 , it does show variations in the nucleation order, $b$, and the nucleation rate constant, $k_{\mathrm{b}}$, implying that the sensitivity of the nucleation detecting instrument is exhibited in the nucleation kinetic parameters rather than the growth parameters. An explanation for this observed effect could lie in the relationship between the true nucleation point and the apparent nucleation point. The most basic interpretation of the maximum sub-cooling (method 1) assumes that the nuclei are formed at their detectable sizes at the time, $t_{\text {det }}$, when they are being detected - quite a feat given the detected crystals are of a relatively large size $(\approx 14 \mu \mathrm{m})$. Method 2 attempts to address this shortfall through the addition of the minimum detectable number density, $N_{\mathrm{m}} / V$, in addition to the same basic assumptions. Method 3 avoids these assumptions by considering the nuclei forming at their undetectable critical size at time $t$, before growing to a detectable size at time, $t_{\mathrm{det}}$. Obviously, the larger the minimum detectable size by the nucleation monitoring device, the longer the growth period from $t$ to $t_{\mathrm{det}}$. This extended growth period is in turn reflected in the larger nucleation order, $b$, predicted for the MGV
1 
Table 2 Results of parameter estimation using outline methods

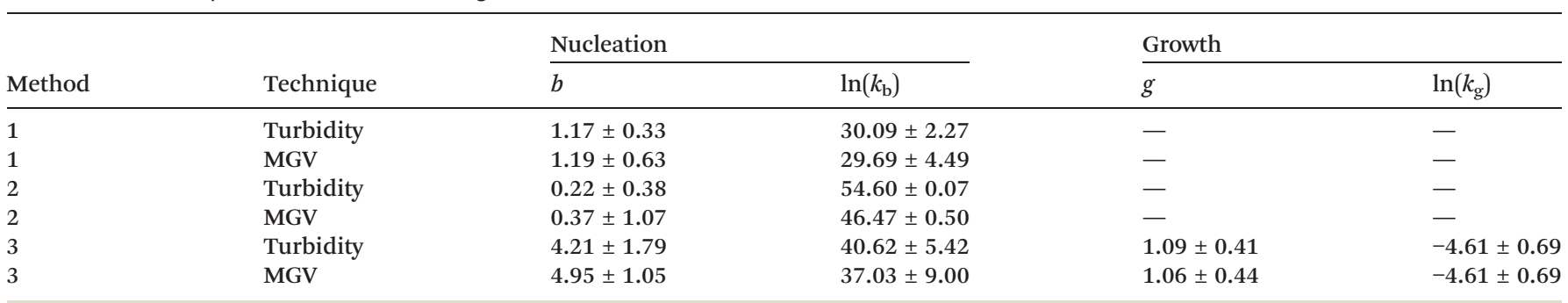

measurements when compared to the turbidity measurements in Table 2. Effectively, this hypothesis implies that the former technique detects a higher density of nuclei than the latter one, with more growth activity occurring between $t$ and $t_{\mathrm{det}}$ in the former than in the latter case. In terms of growth kinetics, derived growth constants by method 3 are of the same order of magnitude, ${ }^{29}$ suggesting similar growth kinetics from the detectable nuclei to the final crystal sizes for both methodologies. In addition, method 3 provides good estimates of growth kinetics in comparison to a more complex model (i.e. one which considered secondary nucleation and agglomeration ${ }^{29}$ ). The authors would like to note that the small variance in nucleation parameters has been shown for the comparison of only two nucleation detection devices. Further experiments covering a range of detection devices would be required to validate this hypothesis.

The predicted maximum sub-cooling for each method and the average absolute relative error (experimental vs. predicted) is shown in Table 3 . All 6 possible combinations of modelling methods and detecting techniques give a reasonable predication of the obtainable maximum sub-cooling when compared to the experimental data (Table 1) via the average absolute relative error (lower percentage indicates smaller deviation between predicted and experimental values). If the traditional Nývlt approach (method 1) is considered as a benchmark for the predictive accuracy, the detectable number density proposed by Kubota (method 2) improves the error between the predicted and experimental measurements for both the turbidity and MGV techniques by an average of $6.8 \%$. For methods $1 \& 2$, the greatest contributions to the residuals between the predicted and experimental values are from data at faster cooling rates, i.e. $1.67^{\circ} \mathrm{C}$ $\min ^{-1}$, where the absolute relative error can be an order of magnitude larger in comparison to that at slower cooling rates.

Building upon this increasing complexity, the consideration of both nucleation and growth rates implemented in method 3 further reduces this error by an average of $94 \%$ in comparison to the baseline (method 1 ). The cause of this can be related back to the derived expressions for methods 1 and 2 in eqn (2) and (5) respectively. In these expressions there is a proposed linear relationship between the cooling rate, $\beta$, and the maximum sub-cooling, $\Delta T_{\max }$, however, the experimental results shown in Table 1 suggest that this linear trend is only reliable over a small range of slower cooling rates, e.g. from 0.1 to $0.64{ }^{\circ} \mathrm{C} \mathrm{min}^{-1}$, while the larger relative error is generated at faster cooling rates. In contrast, the population balance (method 3 ) is not bound by a linear relationship allowing it to match more closely the nonlinear trends of $\beta v s . \Delta T_{\max }$ over a wider range of cooling rates.

From the experimental data of $\Delta T_{\max }$ in Table 1 , the predicted values in Table 3 also demonstrate lower maximum sub-coolings for the turbidity in comparison to that for the MGV measurements (not surprising since these are derived from the same experimental data). Furthermore, when comparing the predicted values for each detection technique with the experimental data, we see that the average absolute relative error for the turbidity technique is universally lower across all three models. In addition, the error diminishes as the complexity of kinetic model increases. This would suggest that not only does the population balance approach predict maximum sub-coolings which are closer to the experimental data, but also the effect of the device utilized to detect nucleation on the predictions is less significant.

For completeness, Table 4 compiles the measured and predicted characteristic length of crystals in this study;

\begin{tabular}{|c|c|c|c|c|c|c|}
\hline \multirow[b]{2}{*}{$\beta$} & \multicolumn{2}{|l|}{ Method 1} & \multicolumn{2}{|l|}{ Method 2} & \multicolumn{2}{|l|}{ Method 3} \\
\hline & $\Delta T_{\max }$ & $\underline{\Delta T_{\max }}$ & $\Delta T_{\max }$ & $\underline{\Delta T_{\max }}$ & $\underline{\Delta T_{\max }}$ & $\underline{\Delta T_{\max }}$ \\
\hline 1.67 & 7.14 & 10.59 & 6.77 & 8.98 & 5.62 & 7.85 \\
\hline 0.64 & 3.15 & 4.73 & 3.08 & 4.45 & 4.05 & 5.48 \\
\hline 0.44 & 2.28 & 3.46 & 2.27 & 3.39 & 2.49 & 4.32 \\
\hline 0.10 & 0.62 & 0.96 & 0.65 & 1.11 & 0.66 & 1.36 \\
\hline Average absolute relative error & $13.1 \%$ & $26.7 \%$ & $12.3 \%$ & $24.8 \%$ & $0.7 \%$ & $1.6 \%$ \\
\hline
\end{tabular}


Table 4 Experimental and predicted crystal size at point of detection using method 3

\begin{tabular}{|c|c|c|c|c|}
\hline & & Experimental & Turbidity & MGV \\
\hline Exp & $\beta\left({ }^{\circ} \mathrm{C} \min ^{-1}\right)$ & $L_{\mathrm{d}}(\mu \mathrm{m})$ & $L_{\mathrm{d}}(\mu \mathrm{m})$ & $L_{\mathrm{d}}(\mu \mathrm{m})$ \\
\hline A & 1.67 & $14.41 \pm 12.50$ & 44.27 & 58.09 \\
\hline B & 0.64 & $15.65 \pm 17.91$ & 69.64 & 76.74 \\
\hline $\mathrm{C}$ & 0.44 & $28.98 \pm 40.56$ & 65.48 & 69.47 \\
\hline D & 0.22 & $13.37 \pm 21.41$ & 18.18 & 97.75 \\
\hline $\mathrm{E}$ & 0.23 & $14.41 \pm 12.61$ & 23.82 & 26.41 \\
\hline $\mathbf{F}$ & 0.10 & $14.88 \pm 12.80$ & 15.45 & 44.02 \\
\hline
\end{tabular}

clearly the predicted values for both detecting tools are much larger than the measured ones, but with closer predictions for lower cooling rates, $\beta$, (see Table 4) than for the higher rates. The reason for this may be due to the complication and interlink of the primary nucleation and growth rates, $B$ and $G$, respectively. The growth of the characteristic length of crystals predicted by the models depends on the optimized kinetic parameters $k_{\mathrm{g}}$ and $k_{\mathrm{b}}$ within the tolerance range, as shown in Table 2, where nucleation occurs. This range varies more for higher cooling rates.

\section{Conclusions}

In summary the work presented here shows for the first time the estimations of the nucleation kinetic parameters, $k_{\mathrm{b}}$ and $b$, for crystallization of adipic acid in an oscillatory baffled crystallizer. The nucleation rate constant, $\ln \left(k_{\mathrm{b}}\right)$, was found to range from $29.69 \pm 4.49$ to $54.60 \pm 0.07$, whereas the nucleation order, $b$, from $0.22 \pm 0.38$ to $4.95 \pm 1.05$, depending on the interpretations of the maximum sub-cooling, $\Delta T_{\max }$, implemented.

For the Nývlt interpretation, the modelled maximum supercoolings have on average a $19.9 \%$ absolute relative error in comparison to the experimental data with the closer predictions at lower cooling rates. The modelled nucleation kinetics are very similar for both turbidity and MGV methods. The Kubota interpretation reduces this error to $18.6 \%$ because of its consideration of the sensitivity of detection device. Precisely due to the inclusion of such a sensitivity, there is a significant difference between the modelled nucleation parameters for the turbidity and MGV techniques The implementation of the population balance model approach further reduces this error to $1.2 \%$ when compared to the experimental data of the maximum supercoolings, ranking this model the most accurate. The model also predicts both nucleation and growth kinetics parameters with very comparable values for both turbidity and MGV techniques. In addition, the influence of the sensitivity of detection device is exhibited in the nucleation order, $b$.

Due to their linear assumptions, both the Nývlt and the Kubota interpretations are found to be most accurate over a narrower range $\left(0.1\right.$ to $\left.0.64{ }^{\circ} \mathrm{C} \mathrm{min}^{-1}\right)$ of cooling rates, whereas the nonlinear nature of the population balance approach makes it accurate over the whole range (0.1 to $1.67^{\circ} \mathrm{C} \mathrm{min}^{-1}$ ) of experiments.

\section{Appendix 1}

Calculating detectable number density, $N_{\mathrm{m}} / V$

To evaluate $N_{\mathrm{m}} / V$ for the MGV technique, the volume, $V$, of the focused area was measured to be $11.37 \mathrm{~mm} \times 12.52 \mathrm{~mm}$, the thickness of the focused area was assumed to be equal to the aperture size of the laser, $2.31 \mathrm{~mm}$, resulting in a sampling volume of $3.29 \times 10^{-7} \mathrm{~m}^{3}$. For the minimum detectable number, $N_{\mathrm{m}}$, manual inspection of the images taken at the point of nucleation counted $17 \pm 5$ crystals averaged over all trials. Therefore the minimum detectable number density, $N_{\mathrm{m}} / V$, was calculated to be $5.17 \times 10^{7} \# \mathrm{~m}^{-3}$. Detectable number density for the turbidity probe was taken to be $1 \times 10^{11} \# \mathrm{~m}^{-3}$ from the work of Mitchell and Frawley. ${ }^{30}$

\section{Acknowledgements}

The authors would like to thank EPSRC and the EPSRC Centre for Innovative Manufacturing in Continuous Manufacturing and Crystallisation for funding this work.

\section{Notes and references}

1 N. Kubota, J. Cryst. Growth, 2008, 310, 629-634.

2 J. Nývlt, J. Cryst. Growth, 1968, 3-4, 377-383.

3 K. Sangwal, Cryst. Res. Technol., 2009, 44, 231-247.

4 K. Sangwal and K. Wójcik, Cryst. Res. Technol., 2009, 44, 363-372.

5 D. Kashchiev, A. Borissova, R. B. Hammond and K. J. Roberts, J. Phys. Chem. B, 2010, 114, 5441-5446.

6 M. Fujiwara, P. S. Chow, D. L. Ma and R. D. Braatz, Cryst. Growth Des., 2002, 2, 363-370.

7 L. L. Simon, Z. K. Nagy and K. Hungerbuhler, Chem. Eng. Sci., 2009, 64, 3344-3351.

8 L. L. Simon, Z. K. Nagy and K. Hungerbuhler, Org. Process Res. Dev., 2009, 13, 1254-1261.

9 F. J. Kumar, S. G. Moorthy, D. Jayaraman and C. Subramanian, J. Cryst. Growth, 1996, 160, 129-135.

10 A. R. Parsons, S. N. Black and R. Colling, Chem. Eng. Res. Des., 2003, 81, 700-704.

11 O. J. Joung, Y. H. Kim and K. Fukui, Sens. Actuators, B, 2005, 105, 464-472.

12 H. Gürbüz and B. Özdemir, J. Cryst. Growth, 2003, 252, 343-349.

13 P. Barrett and B. Glennon, Chem. Eng. Res. Des., 2002, 80, 799-805.

14 N. Gherras and G. Fevotte, J. Cryst. Growth, 2012, 342, 88-98.

15 N. Gherras and G. Fevotte, AIChE J., 2012, 58, 2650-2664.

16 Z. K. Nagy, M. Fujiwara, X. Y. Woo and R. D. Braatz, Ind. Eng. Chem. Res., 2008, 47, 1245-1252.

17 C. J. Brown and X.-W. Ni, Cryst. Growth Des., 2011, 11, 3994-4000.

18 C. J. Callahan and X.-W. Ni, Cryst. Growth Des., 2012, 12, 2525-2532.

19 C. J. Callahan and X.-W. Ni, CrystEngComm, 2014, 16, 690-697. 
20 M. Kobari, N. Kubota and I. Hirasawa, J. Cryst. Growth, 2011, 317, 64-69.

21 A. D. Randolph and M. A. Larson, Theory of particulate processes: analysis and techniques of continuous crystallization, Academic Press, 1988.

22 CSID:191, http://www.chemspider.com/Chemical-Structure.191.html, (accessed 14:21, Jan 13, 2014).

23 C. B. B. Costa, A. C. da Costa and R. M. Filho, Chem. Eng. Process., 2005, 44, 737-753.

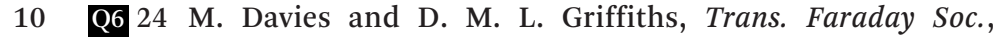
1953, 49.
25 J. W. Mullin, Crystallisation, Butterworth-Heinmann, Oxford, UK, 2001.

26 P. H. Gaskell, Y. C. Lee and H. M. Thompson, Comput. Model. Eng. Sci., 2010, 62, 77-112.

27 C. J. Brown and X.-W. Ni, CrystEngComm, 2012, 14, 2944-2949. 5

28 L. L. Simon, K. A. Oucherif, Z. K. Nagy and K. Hungerbuhler, Ind. Eng. Chem. Res., 2010, 49, 9932-9944.

29 R. David, J. Villermaux, P. Marchal and J.-P. Klein, Chem. Eng. Sci., 1991, 46, 1129-1136.

30 N. A. Mitchell and P. J. Frawley, J. Cryst. Growth, 2010, 312, 2740-2746. 